\title{
Low-strain $\mathrm{Si} / \mathrm{O}$ superlattices with tunable electronic properties: $A \boldsymbol{b}$ initio calculations
}

\author{
Kengo Nishio, ${ }^{1, *}$ Anh Khoa Augustin Lu, ${ }^{2,3}$ and Geoffrey Pourtois ${ }^{2,4}$ \\ ${ }^{1}$ National Institute of Advanced Industrial Science and Technology (AIST), Central 2, Umezono 1-1-1, Tsukuba, \\ Ibaraki 305-8568, Japan \\ ${ }^{2}$ IMEC, 75 Kapeldreef, B-3001 Leuven, Belgium \\ ${ }^{3}$ Department of Physics, Katholieke Universiteit Leuven, B-3001 Leuven, Belgium \\ ${ }^{4}$ PLASMANT, Department of Chemistry, University of Antwerp, B-2610 Antwerp, Belgium \\ (Received 26 May 2014; revised manuscript received 6 March 2015; published 16 April 2015)
}

\begin{abstract}
We propose that low-strain $\mathrm{Si} / \mathrm{O}$ superlattices can be constructed by connecting reconstructed $\mathrm{Si}\{001\}$ surfaces by $\mathrm{Si}-\mathrm{O}-\mathrm{Si}$ bridges. Ab initio calculations show that our models are energetically more favorable than all the models proposed so far. The part of our $\mathrm{Si} / \mathrm{O}$ superlattice model is experimentally accessible just by oxidizing a $\mathrm{Si}(001)$ substrate. To complete our $\mathrm{Si} / \mathrm{O}$ superlattice model, we propose a three-step method. We also explore the potential of our $\mathrm{Si} / \mathrm{O}$ superlattice models for new materials used in future Si electronics. We find that the location of the channel where the carriers travel can be controlled between the interfaces and the Si layers by the insertion of $\mathrm{O}$ atoms into the $\mathrm{Si}$-Si dimers. By revealing the origins of the interface electron and hole states, we find that similar interface states should be easily achieved for Si slabs and Si substrates. Interestingly, the interface electrons and holes have small effective masses in the direction parallel to the channel and large effective masses in the direction normal to the channel, which makes the Si/O superlattices attractive to be used for channel materials. We also find that the valley splitting of $\mathrm{Si}$ is enhanced by the formation of the $\mathrm{Si} / \mathrm{O} / \mathrm{Si}$ interfaces, which is ideal for developing Si-based qubits. Our findings open new perspectives to design and control the electronic properties of Si.
\end{abstract}

DOI: 10.1103/PhysRevB.91.165303

PACS number(s): 68.65.Cd, 68.35.-p, 73.20.At, 73.21.Cd

\section{INTRODUCTION}

Challenges to explore new materials and phenomena are the driving forces to further extend the frontiers of materials science and technology. For instance, for Si-based materials, the discovery of photoluminescence from Si nanostructures has stimulated studies of the impact of quantum confinement effects to tune the light-emitting properties of $\mathrm{Si}$ and to realize Si-based optoelectronics [1-10]. Another example is the discovery of piezoresistance in $\mathrm{Si}$ and Ge [11], which has stimulated the investigation of the effects of strain on the electronic transport properties of Si [12-15]. Nowadays, strained $\mathrm{Si}$ is commonly used to enhance the performance of Si-based transistors [13]. Recently, the impact of computer simulations to offer guidelines in the identification of potentially useful new materials has been increasing [14-21]. For example, silicene was first predicted by ab initio calculations [19] and then later on realized experimentally [20,21].

The idea of exploiting semiconductor atomic superlattices (SLs), which are composed of alternate epitaxial semiconductor layers and atomically thin layers of adsorbed atoms, was proposed by Tsu to control quantum confinement effects [22-24]. Recently, the Si/O SL concept has been gaining momentum and is expected to provide a new opportunity to improve the electronic transport properties of $\mathrm{Si}[25,26]$. The epitaxial growth of $\mathrm{Si}$ on an $\mathrm{O}$ monolayer adsorbed on a $\mathrm{Si}(001)$ substrate and the resulting epitaxial $\mathrm{Si} / \mathrm{O}$ SLs have been demonstrated [24-26]. However, given their complex nature, the atomic structure of the $\mathrm{Si} / \mathrm{O} / \mathrm{Si}$ interfaces has not been thoroughly resolved experimentally and remains a mystery. The identification of the most stable structures of the

*k-nishio@aist.go.jp
$\mathrm{Si} / \mathrm{O} / \mathrm{Si}$ interface would be valuable for optimizing the growth conditions and tailoring their electronic transport properties. Several structure models of the Si/O SL have already been proposed [23,27]. However, these have large formation energies and suffer from large strains as discussed later.

In this paper, we theoretically explore the most stable structures of the Si/O SL and propose a method to realize them experimentally. Our ab initio calculations show that low-energy and low-strain Si/O SLs can be constructed by connecting reconstructed $\mathrm{Si}\{001\}$ surfaces [28] by Si-O-Si bridges. The part of our Si/O SL model has already been realized experimentally. By extending the technique, we propose a three-step method to complete our Si/O SL model. We also explore the potential of our Si/O SL models for new materials used in future $\mathrm{Si}$ electronics. Interestingly, our $\mathrm{Si} / \mathrm{O} \mathrm{SL}$ models have unique electronic properties such as the tunable channel location, small carrier effective masses, and large valley splitting, which makes them attractive materials for advanced Si electronics.

\section{LOW-STRAIN STRUCTURE}

\section{A. Geometrical consideration}

Our strategy for constructing the Si/O SL models consists in connecting the reconstructed $\operatorname{Si}\{001\}$ surfaces by the Si-O-Si bridges, as illustrated in Fig. 1(a) (Rec-I interface). This $\mathrm{Si} / \mathrm{O} / \mathrm{Si}$ interface has the following five geometrical features, which allow each atom to maintain its natural bonding configuration.

(i) The arrangement of the $\mathrm{Si}$ atoms involved in the $\mathrm{Si} / \mathrm{O} / \mathrm{Si}$ interface is intrinsic to $\mathrm{Si}$, so that the interface does not induce a large strain in the Si layers.

(ii) All the dangling bonds of the Si-Si dimers are passivated by the Si-O-Si bridging bonds. 
(a) Rec-I

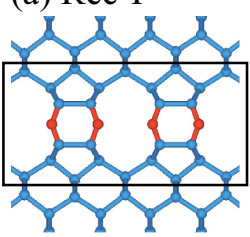

(c) Luo-I

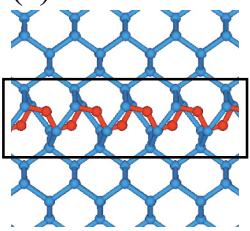

(b) Rec-II

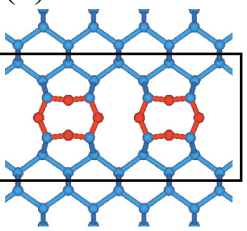

(d) Tsu-II

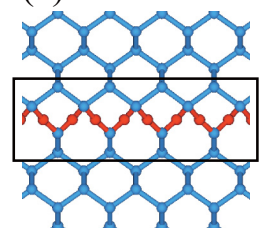

(e) Rec-I-20ML

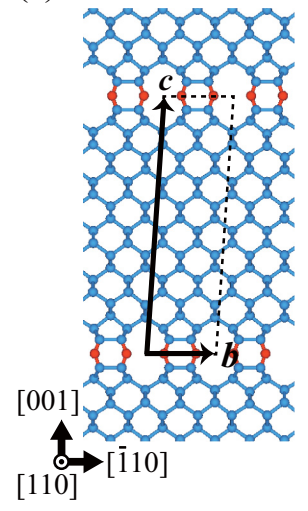

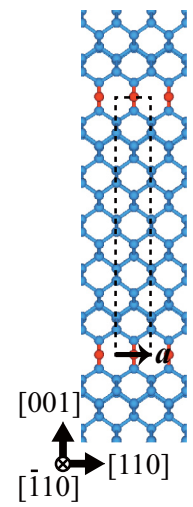

$\boldsymbol{a}=\left(l_{\mathrm{b}} / 2, l_{\mathrm{b}} / 2,0\right)$

$\boldsymbol{b}=\left(-l_{\mathrm{b}}, l_{\mathrm{b}}, 0\right)$

$c=\left(c_{x}=-c_{y}, c_{y}, c_{z}\right)$

(f) Rec-I-4ML

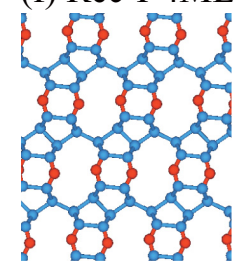

FIG. 1. (Color online) Atomic arrangement. (a) Rec-I, (b) Rec-II, (c) Luo-I, and (d) Tsu-II Si/O/Si interface models. Blue and red (light and dark gray) particles are $\mathrm{Si}$ and $\mathrm{O}$ atoms, respectively. Blue and red (light and dark gray) bonds are $\mathrm{Si}-\mathrm{Si}$ and $\mathrm{Si}-\mathrm{O}$ bonds, respectively. Framed regions define the $\mathrm{Si} / \mathrm{O} / \mathrm{Si}$ interfaces to evaluate the lattice mismatches of the interfaces with Si. (e) Epitaxial Rec-I-20ML SL. The dashed lines show the unit cell. The arrows are the unit-cell vectors: $\boldsymbol{a}, \boldsymbol{b}$, and $\boldsymbol{c}$. (f) Freestanding Rec-I-4ML SL.

(iii) The $\mathrm{Si}-\mathrm{O}$ bond lengths and $\mathrm{O}-\mathrm{Si}-\mathrm{Si}$ bond angles can be tuned to their optimal values by adjusting the positions of the $\mathrm{O}$ atoms and the distance between the $\mathrm{Si}$ surfaces.

(iv) The Si-O-Si bond angles can adapt themselves to a geometry determined by the optimal Si-O bond lengths and the $\mathrm{O}-\mathrm{Si}-\mathrm{Si}$ bond angles because of the flexibility of the $\mathrm{Si}-\mathrm{O}-\mathrm{Si}$ bridging bonds.

(v) The oxidation state of the interface $\mathrm{Si}$ atoms can be tuned by inserting additional $\mathrm{O}$ atoms into the $\mathrm{Si}$-Si dimers, as illustrated in Fig. 1(b) (Rec-II interface), without inducing a large strain since the oxygen inserted dimers ( $\mathrm{Si}-\mathrm{O}-\mathrm{Si}$ dimers) are energetically favorable structures [29].

For comparison purpose, interface models proposed by Luo et al. [27] and Tsu et al. [23], hereafter referred to as the Luo-I and Tsu-II models, are illustrated in Figs. 1(c) and 1(d), respectively. Here, I (II) indicates the oxidation state of the forefront $\mathrm{Si}$ atoms $+1(+2)$. Note that the area densities of the $\mathrm{O}$ atoms for the Rec-I and Luo-I (Rec-II and Tsu-II) are 2 (4) atoms per $l_{b}^{2}$, where $l_{b}$ is the lattice constant of bulk Si: $5.460 \AA$ in our simulation scheme (see the Supplemental Material for details [30]).

\section{B. $A b$ initio calculation}

To qualitatively investigate the stabilities of the SLs, we carried out $a b$ initio calculations using the QMAS code [31]. The QMAS code is based on the density functional theory (DFT) with the generalized gradient approximation (GGA) [32] and the projector-augmented-wave method [33]. The planewave cut-off energy was set to $50 \mathrm{Ry}$. The convergence criteria of $5 \times 10^{-5}$ Hartree/Bohr for forces on atoms and $3 \times 10^{-7}$ Hartree $/$ Bohr $^{3}$ for the stress components were used. $8 \times 4 \times 4,8 \times 4 \times 2$, and $8 \times 4 \times 2 k$-point grids were used for $4 \mathrm{ML}, 20 \mathrm{ML}$, and $40 \mathrm{ML}$ SLs, respectively.

We first optimized the structures of the epitaxial RecI-20ML [Fig. 1(e)], Rec-II-20ML, Luo-I-20ML, and TsuII-20ML SLs. Here, $20 \mathrm{ML}$ indicates the number of $\mathrm{Si}$ monolayers (MLs) in each Si layer. Although the interface between the Si substrate and the $\mathrm{Si} / \mathrm{O} \mathrm{SL}$ was not included in a unit cell, the in-plane unit-cell vectors $\boldsymbol{a}$ and $\boldsymbol{b}$ of the epitaxial $\mathrm{Si} / \mathrm{O} \mathrm{SL}$ were constrained to match the $\mathrm{Si}(001)$ substrate (see the Supplemental Material for details [30]). On the other hand, the out-of-plane vector $c$ and all the atomic positions were optimized to minimize the total energy. Note that the $a$ and $b$ axes were set to be along the [110] direction normal to the $\mathrm{Si}-\mathrm{Si}$ dimers and the [110] direction parallel to the $\mathrm{Si}-\mathrm{Si}$ dimers, respectively.

We compare the stabilities of the Si/O SLs in terms of their formation energies per $\mathrm{O}$ atom:

$$
f \equiv\left(E_{\mathrm{tot}}-n_{\mathrm{S} i} \mu_{\mathrm{S} i}-n_{\mathrm{O}} \mu_{\mathrm{O}}\right) / n_{\mathrm{O}} .
$$

Here, $E_{\text {tot }}$ is the total energy per unit cell containing $n_{\mathrm{Si}} \mathrm{Si}$ atoms and $n_{\mathrm{O}} \mathrm{O}$ atoms. The chemical potentials $\mu_{\mathrm{S} i}$ and $\mu_{\mathrm{O}}$ correspond to the energies per atom calculated for bulk $\mathrm{Si}$ and for the $\mathrm{O}_{2}$ molecules, respectively. The results summarized in Table I demonstrate that our Rec-I-20ML (Rec-II-20ML) model is more stable by $0.60(1.05) \mathrm{eV} /$ atom than the Luo-I20ML (Tsu-II-20ML) model.

To further demonstrate the advantages of our models, we investigate the strain present in the Si/O SLs. The lattice mismatches are often used as a measure of the strain in a SL composed of materials having the same crystal structure.

TABLE I. Formation energies and lattice mismatches calculated for the different models. $\epsilon_{a} \equiv\left(a-a_{\mathrm{b}}\right) / a_{\mathrm{b}} . a$ and $b$ are the lengths of the in-plane cell vectors. $\gamma$ is the angle made between $\boldsymbol{a}$ and $\boldsymbol{b} . a_{\mathrm{b}}, b_{\mathrm{b}}$, and $\gamma_{\mathrm{b}}$ are the values for bulk Si: $\sqrt{2} l_{\mathrm{b}} / 2, \sqrt{2} l_{\mathrm{b}}$, and $90^{\circ}$, respectively.

\begin{tabular}{lcccc}
\hline \hline & $f(\mathrm{eV} /$ atom $)$ & $\epsilon_{a}(\%)$ & $\epsilon_{b}(\%)$ & $\epsilon_{\gamma}(\%)$ \\
\hline epitaxial Rec-I-20ML & -3.62 & - & - & - \\
epitaxial Luo-I-20ML & -3.02 & - & - & - \\
epitaxial Rec-II-20ML & -4.08 & - & - & - \\
epitaxial Tsu-II-20ML & -3.03 & - & - & - \\
freestanding Rec-I-4ML & -3.67 & 0.0 & -1.5 & 0.0 \\
freestanding Luo-I-4ML & -3.19 & -0.2 & 8.6 & 0.0 \\
freestanding Rec-II-4ML & -4.09 & 2.5 & 2.7 & 0.0 \\
freestanding Tsu-II-4ML & -3.52 & 4.9 & 4.9 & -18.6 \\
\hline \hline
\end{tabular}


For $\mathrm{Si} / \mathrm{O} \mathrm{SLs}$, however, the lattice mismatches are not well defined because the atomically thin $\mathrm{O}$ layer does not have a corresponding bulk structure. Accordingly, instead of calculating the lattice mismatches of $\mathrm{O}$ with $\mathrm{Si}$, we calculated those of the $\mathrm{Si} / \mathrm{O} / \mathrm{Si}$ interfaces with $\mathrm{Si}$. For this purpose, we define the $\mathrm{Si} / \mathrm{O} / \mathrm{Si}$ interfaces as the framed regions illustrated in Figs. 1(a)-1(d). The lattice mismatches of the $\mathrm{Si} / \mathrm{O} / \mathrm{Si}$ interface can be captured by its corresponding $4 \mathrm{ML}$ SL constructed by stacking the interfaces. To quantify the lattice mismatches of the interfaces, the lattice parameters of the freestanding Rec-I-4ML [Fig. 1(f)], Rec-II-4ML, Luo-I-4ML, and Tsu-II-4ML SLs were calculated by optimizing all the unit-cell vectors without applying any constraint. Note that freestanding indicates that the SL is not grown on a Si substrate. The results are summarized in Table I together with their corresponding formation energies. The lattice mismatches of the Rec-I and Rec-II interfaces are at most $2.7 \%$. On the other hand, the Luo-I interface has a mismatch of $8.6 \%$ along the $b$ direction. This originates from the fact that the $\mathrm{O}$ atoms are forced into $\mathrm{Si}-\mathrm{Si}$ bonds of the diamond structure. The Tsu-II interface has a mismatch of $-18.6 \%$ in the angle made between the $\boldsymbol{a}$ and $\boldsymbol{b}$ unit-cell vectors. This large distortion originates from the fact that the O-Si-O bond of the interface $\mathrm{Si}$ atom is twisted around the angle bisector of the $\mathrm{Si}-\mathrm{Si}-\mathrm{Si}$ bond to connect the nonreconstructed $\mathrm{Si}\{001\}$ surfaces by the $\mathrm{Si}-\mathrm{O}-\mathrm{Si}$ bridges. These results demonstrate that our $\mathrm{Si} / \mathrm{O} / \mathrm{Si}$ interfaces offer better epitaxial matches with $\mathrm{Si}$ than the interfaces proposed so far.

\section{Clue to the experimental realization}

The part of the Rec-II interface is experimentally accessible [34-37]. Actually, when a $\mathrm{Si}(001)$ substrate is oxidized, an atomically thin tridymite $\left(\mathrm{t}-\mathrm{SiO}_{2}\right)$ layer can form between the $\mathrm{Si}$ and amorphous $\mathrm{SiO}_{2}\left(\mathrm{a}-\mathrm{SiO}_{2}\right)$. The structure of the $\mathrm{Si} / \mathrm{t}-\mathrm{SiO}_{2}$ interface resembles to that of the Rec-II interface in the sense that it contains the Si-O-Si dimers and that the dangling bonds of the dimers are passivated by the $\mathrm{Si}-\mathrm{O}-\mathrm{Si}$ bridging bonds. Note that although a $\mathrm{Si} / \mathrm{t}-\mathrm{SiO}_{2}$ structure model composed of $\mathrm{Si}-\mathrm{Si}$ dimers were considered in the original study of Ourmazd [34], the Si-O-Si dimers would be energetically more favorable [29,35-37]. The whole Rec-II interface should be obtained by a three-step method as follows:

(i) oxidation of a $\mathrm{Si}(001)$ to form the $\mathrm{Si} / \mathrm{t}-\mathrm{SiO}_{2} / \mathrm{a}-\mathrm{SiO}_{2}$ structure,

(ii) hydrofluoric acid treatment to remove needless $\mathrm{t}-\mathrm{SiO}_{2}$ and $\mathrm{a}-\mathrm{SiO}_{2}$,

(iii) epitaxial growth of Si to complete the Rec-II interface. Finally, the Rec-II SL can be obtained by repeating the threestep method. Note that in step (i) the thickness of $\mathrm{SiO}_{2}$ layer may be important because it is proposed that a $\mathrm{Si} /$ cristobalite $\left(\mathrm{c}-\mathrm{SiO}_{2}\right)$ interface forms at the initial stage of oxidation and the $\mathrm{c}-\mathrm{SiO}_{2}$ layer transforms to the $\mathrm{t}-\mathrm{SiO}_{2}$ layer to release compressive strains as the oxidation proceeds $[34,37,38]$.

In addition to the epitaxial growth, our $\mathrm{Si} / \mathrm{O} \mathrm{SL}$ models should be accessible by a solution-phase synthesis using hydrolysis of $\mathrm{OH}$-terminated Si membranes [39] because our $\mathrm{Si} / \mathrm{O}$ SLs are energetically more favorable without the $\mathrm{Si}(001)$ substrate than the SLs proposed so far (Table I).

\section{UNIQUE ELECTRONIC PROPERTIES}

To explore the unique electronic properties, we calculated the electronic states of the epitaxial Rec-I and Rec-II SLs using the OpenMX code [40]. The OpenMX code is based on the DFT with the GGA [32] and the norm-conserving pseudopotentials [41]. The wave functions (WFs) are expressed by the linear combination of pseudoatomic orbitals. The basis sets, Si7.0-s3p3d2, O5.0-s3p3d2, and H5.0-s3p3 were used for $\mathrm{Si}, \mathrm{O}$, and $\mathrm{H}$ atoms, respectively, except for the 40ML SLs where Si7.0-s $2 p 2 d 1$ and O5.0-s $2 p 2 d 1$ were used. Here, the abbreviation, for example Si7.0-s3p3d2, indicates that three $s$, three $p$, and two $d$ orbitals of a $\mathrm{Si}$ atom with the cut-off radius of 7.0 Bohr were employed. The effective masses were calculated from the band structures obtained by a noncollinear DFT including the spin-orbit coupling [42-44], while the WFs were calculated by a spin-unpolarized scheme. $16 \times 8 \times 8$, $16 \times 8 \times 2$, and $16 \times 8 \times 2 k$-point grids were used for $4 \mathrm{ML}$, 20ML and 40ML Si/O SLs, respectively.

\section{A. Tunability of the location of the channel}

Figure 2 shows the band structures and isosurfaces of the WFs of our SLs. For comparison, we provide the corresponding band structure and WFs of bulk Si in Appendix A. The most remarkable feature of our SLs is the tunability of the location of the channel where the electrons or holes travel. The WF of the $\mathrm{C}_{\mathrm{I}}$ level of the Rec-I-20ML SL, which is the bottom of the first lowest conduction band (CB1), is localized around the $\mathrm{Si} / \mathrm{O} / \mathrm{Si}$ interfaces. On the other hand, the WF of the $\mathrm{V}_{a}$ level, which is the top of the first highest valence band (VB1), delocalizes over the Si layers. To our surprise, the situation is reversed in the Rec-II-20ML SL. The VB1-top $V_{\text {II }}$ level is the interface state, while the WF of the CB1-bottom $\Delta_{2 \Gamma}$ level delocalizes over the Si layers. The same behaviors are also found in the Rec-I- and Rec-II-40ML SLs (Appendix B). We also show the WFs of a Si/O SL composed of alternating Rec-I and Rec-II interfaces in Fig. 2(e). The CB1-bottom WF is localized around the Rec-I interface, while the VB1-top WF is localized around the Rec-II interface. Furthermore, the interface states are also found in isolated slab models (Appendix C). Altogether, the location of the channel can be controlled between the $\mathrm{Si} / \mathrm{O} / \mathrm{Si}$ interfaces and the Si layers by the interface structure.

Note that although the $d$ orbitals are mixed a little, the Rec-I interface state mainly consists of $s, p_{b}$, and $p_{z}$ orbitals. On the other hand, the Rec-II interface state consists of almost $p_{a}$ orbitals only.

\section{B. Origin of interface electron state}

The Rec-I interface electron state primarily originates from the interactions between the adjacent Si layers. This is verified by the fact that the interface electron state also occurs in a pile of Si slabs with H-terminated Si-Si dimers [Fig. 3(a)]. According to a previous study, the energy gap of $\mathrm{SiO}_{2}$ close to the $\mathrm{Si} / \mathrm{SiO}_{2}$ interface is almost the same as the band gap of $\mathrm{Si}$ [36]. Similarly, the $\mathrm{Si} / \mathrm{O} / \mathrm{Si}$ interfaces, corresponding to atomically thin suboxide layers, are not good insulators. Therefore, the WF can penetrate through the $\mathrm{Si} / \mathrm{O} / \mathrm{Si}$ interface, and the electronic states of the adjacent $\mathrm{Si}$ layers can couple each other. 
(a) Rec-I-20ML

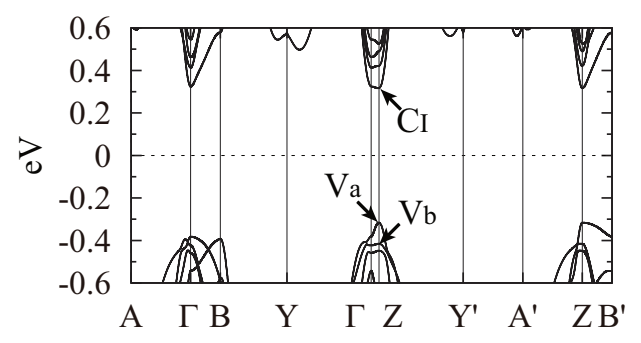

(b) Rec-II-20ML

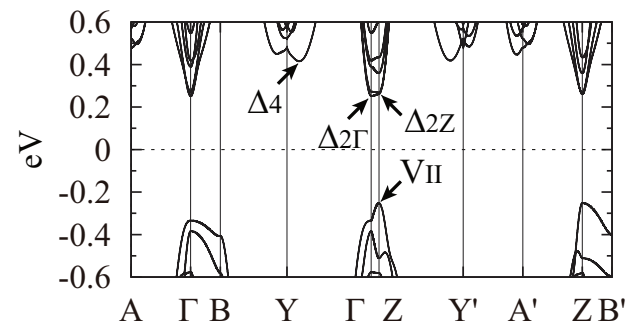

(c) Rec-I-20ML

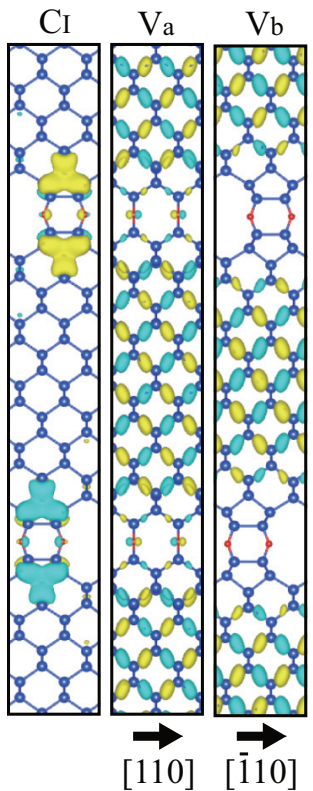

(d) Rec-II-20ML

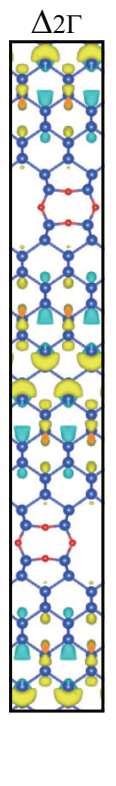

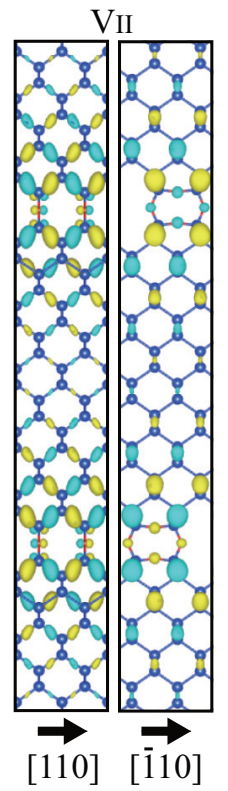

(e) Rec-I\&II-20ML

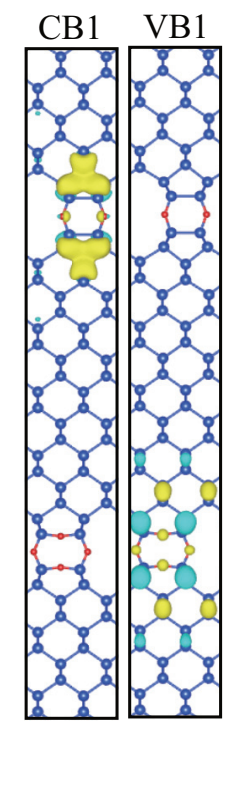

FIG. 2. (Color) Electronic structures of the epitaxial SLs. The band structures of (a) Rec-I- and (b) Rec-II-20ML SLs. Note that the energy is measured from the Fermi level. $\mathrm{A}=\frac{2 \pi}{l_{\mathrm{b}}}\left(\frac{1}{2}, \frac{1}{2}, 0\right) \equiv\left[\frac{1}{2}, \frac{1}{2}, 0\right], \Gamma=[0,0,0], \mathrm{B}=\left[-\frac{1}{4}, \frac{1}{4}, 0\right], \mathrm{Y}=[0,1,0], \mathrm{Z}=\left[0,0, \frac{l_{\mathrm{b}}}{2 c_{z}}\right], \mathrm{Y}^{\prime}=\left[0,1, \frac{l_{\mathrm{b}}}{2 c_{z}}\right]$, $\mathrm{A}^{\prime}=\left[\frac{1}{2}, \frac{1}{2}, \frac{l_{\mathrm{b}}}{2 c_{z}}\right]$, and $\mathrm{B}^{\prime}=\left[-\frac{1}{4}, \frac{1}{4}, \frac{l_{\mathrm{b}}}{2 c_{z}}\right]$. These points are not necessarily in the first Brillouin zone. $l_{b}$ and $c_{z}$ are the lattice constant of bulk $\mathrm{Si}$ and $z$ component of out-of-plane unit cell-vector $c$ (See also Fig. 1). (c) The isosurfaces $\left( \pm 0.020\right.$ in units of electron $\left.{ }^{1 / 2} / a_{0}^{3 / 2}\right)$ of WFs of the $\mathrm{C}_{\mathrm{I}}, \mathrm{V}_{\mathrm{a}}$, and $\mathrm{V}_{\mathrm{b}}$ levels of the Rec-I-20ML SL. Here, $a_{0}$ is the Bohr radius. (d) The isosurfaces of WFs of the $\Delta_{2 \Gamma}$ level ( \pm 0.018 ) and $\mathrm{V}_{\mathrm{II}}$ level $( \pm 0.020)$ of the Rec-II-20ML SL. (e) The isosurfaces $\left( \pm 0.020 \times 2^{1 / 2}\right)$ of the CB1-bottom and VB1-top WFs of a SL composed of alternating Rec-I and Rec-II interfaces. Note that the isosurface level is $2^{1 / 2}$ times larger than that used in (c) and (d) because the number of Rec-I (Rec-II) interfaces per unit cell is not two but one.

The interface electron state does not occur in the Rec-II SL, and neither in a Rec-I-like SL [Fig. 3(b)] that is constructed by removing $\mathrm{O}$ atoms from the $\mathrm{Si}-\mathrm{O}-\mathrm{Si}$ dimers of the Rec-II SL. The tetrahedral bonds of Si atoms near the Rec-I interface are distorted to form the Si-Si dimers, but the strains in the tetrahedral bonds are partially released when $\mathrm{O}$ atoms are inserted in the Si-Si dimers. This means that the distortion of tetrahedral bonds of Si atoms near the interface has a secondary effect on the formation of the interface state. Note that both the charge transfer induced by the bridging $\mathrm{O}$ atoms and the mixing of the orbitals of the $\mathrm{O}$ atoms are not dominant factors for the formation of the interface state. The role of the $\mathrm{O}$ atoms is just to keep the distance between the Si layers so as to interact with each other.

Interestingly, our finding that the interactions between the adjacent $\mathrm{Si}$ layers induce the interface electron state suggests that a similar interface state should be easily realized experimentally just by facing $\mathrm{H}$-terminated reconstructed Si(001) substrates toward each other.

\section{Origin of interface hole state}

In the Rec-II interface, the $\mathrm{O}$ atoms are inserted in the $\mathrm{Si}-\mathrm{Si}$ dimers. The coupling of the $p_{a}$ orbitals of the inserted $\mathrm{O}$ atoms and those of the Si atoms induces the Rec-II interface hole state. The changes in geometry such as bond length and angle, charge transfer, and orbital coupling are reflected in the $a b$ initio calculations. To evaluate the orbital coupling effect exclusively, we develop a nearest-neighbor tight-binding (TB) model as follows.

Since the tops of the valence bands of both Rec-I and Rec-II SLs are composed of almost $p_{a}$ orbitals only, we ignore the other orbitals, and then expand the WF as

$$
\left|\Psi^{(n \boldsymbol{k})}\right\rangle=\frac{1}{\sqrt{N}} \sum_{i} \sum_{\boldsymbol{R}} C_{i}^{(n \boldsymbol{k})} e^{i \boldsymbol{k} \cdot\left(\boldsymbol{R}+\boldsymbol{r}_{i}\right)}\left|\phi_{\mu(i)}\left(\boldsymbol{R}+\boldsymbol{r}_{i}\right)\right\rangle,
$$

where $n$ is the band index, $\boldsymbol{k}$ is the wave vector, $N$ is the number of atoms in the unit cell, $C_{i}^{(n \boldsymbol{k})}$ is the expansion coefficient, $\boldsymbol{R}$ is the lattice vector, $\boldsymbol{r}_{i}$ is the position vector of the atom $i, \mu(i)$ indicates the atomic species ( $\mathrm{Si}$ or $\mathrm{O}$ ), and $\phi_{\mu(i)}$ indicates the $p_{a}$ orbital of the atom $\mu(i)$.

The links of the interactions are illustrated in Fig. 4. The orbitals of the $\mathrm{Si}$ atoms of the $\mathrm{Si}-\mathrm{Si}$ dimer couple each other. On the other hand, the orbitals of the $\mathrm{Si}$ atoms of the $\mathrm{Si}-\mathrm{O}-\mathrm{Si}$ dimer do not. Instead, those orbitals couple with the orbital of the inserted $\mathrm{O}$ atom. The difference in bond-network topology between the Rec-I and Rec-II SLs is thus taken into account.

The TB parameters are determined to reproduce the band structures calculated with the OpenMX and summarized in Table II. The parameters for $\mathrm{Si}$ are determined to reproduce the band structure of bulk $\mathrm{Si}$ as follows. The value of $E_{p}(\mathrm{Si})$ is set to be $0.0 \mathrm{eV}$. The value of $V_{p p \pi}(\mathrm{Si}-\mathrm{Si})\left[V_{p p \sigma}(\mathrm{Si}-\mathrm{Si})\right]$ is set to reproduce the difference in energy between the $\Gamma_{25}^{\prime}$ and $X_{4}\left(\Gamma_{15}\right.$ and $\left.\Gamma_{25}^{\prime}\right)$ levels [45]. Bulk Si and the Si layer can be regarded as being composed of $a$-directed zigzag chains of $\mathrm{Si}$ atoms. If the nearest-neighbor Si atoms $i$ and $j$ are in the same 
(a)

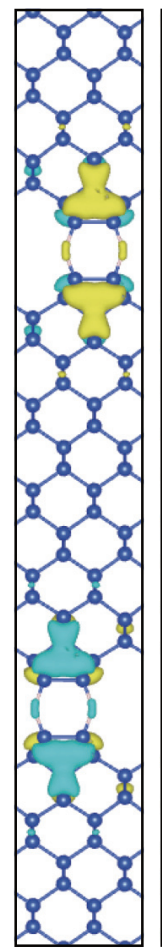

(b)

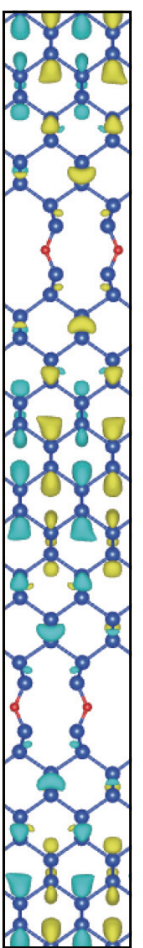

(c)

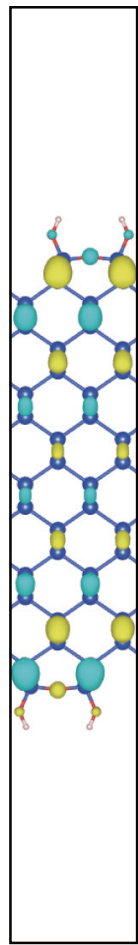

(d)

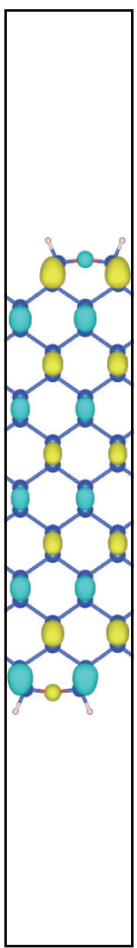

(e)

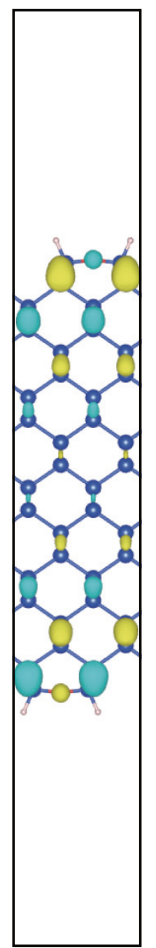

FIG. 3. (Color) Isosurfaces of WFs. (a) CB1-bottom of a pile of Si slabs with H-terminated Si-Si dimers $( \pm 0.020)$. Note that the distance between $\mathrm{H}$ atoms of adjacent $\mathrm{Si}$ slabs is $0.85 \AA$. (b) CB1bottom of the Rec-I-like SL $( \pm 0.020)$. (c) VB1-top of the Si slab with OH-terminated Si-O-Si dimers $\left( \pm 0.020 \times 2^{1 / 2}\right)$. (d) VB1-top of the Si slab with H-terminated Si-O-Si dimers $\left( \pm 0.020 \times 2^{1 / 2}\right)$. (e) VB1-top of the hole-doped Si slab with H-terminated Si-O-Si dimers $\left( \pm 0.020 \times 2^{1 / 2}\right)$. Note that the VB1-top level of the hole-doped $\mathrm{Si}$ slab is not occupied by electrons because holes are doped. The $\mathrm{H}$ atoms are placed $1.48 \AA$ apart from the surface Si atoms or $1.00 \AA$ apart from the $\mathrm{O}$ atoms. The structures are not optimized.

chain, the interaction energy between the $p_{a}$ orbitals for bulk $\mathrm{Si}$ is

$$
\left\langle\phi_{\mathrm{Si}}\left(\boldsymbol{r}_{i}\right)|H| \phi_{\mathrm{Si}}\left(\boldsymbol{r}_{j}\right)\right\rangle=\frac{2 V_{p p \sigma}(\mathrm{Si}-\mathrm{Si})+V_{p p \pi}(\mathrm{Si}-\mathrm{Si})}{3} .
$$

On the other hand, if each nearest-neighbor $\mathrm{Si}$ atom is in a different chain,

$$
\left\langle\phi_{\mathrm{Si}}\left(\boldsymbol{r}_{i}\right)|H| \phi_{\mathrm{Si}}\left(\boldsymbol{r}_{j}\right)\right\rangle=V_{p р \pi}(\mathrm{Si}-\mathrm{Si}) .
$$

We assume the same ones for Si atoms in SLs, whereby the difference in geometry such as bond length and angle is ignored. The value of $E_{p}(\mathrm{O})$ is set to reproduce the difference in energy between the $3 p$ orbital of an isolated $\mathrm{Si}$ atom and the $2 p$ orbital of an isolated $\mathrm{O}$ atom. The value of $V_{p p \pi}^{(\mathrm{I})}(\mathrm{Si}-\mathrm{O})$ is set to reproduce the difference in energy between the $\operatorname{VB} 1(Z)$ and VB1 $(\Gamma)$ levels of the Rec-I-20ML SL. Here, the VB1 $(Z)$ level indicates the level of the VB1 band at the $Z$ point. The value of $V_{p p \pi}^{(\mathrm{II})}(\mathrm{Si}-\mathrm{O})(=-1.488 \mathrm{eV})$, which is determined to reproduce the energy difference for the Rec-II-20ML SL, is different from that of $V_{p р \pi}^{(\mathrm{I})}(\mathrm{Si}-\mathrm{O})(=-1.759 \mathrm{eV})$. This difference primarily originates from the charge transfer. By fixing the value of $V_{p р \pi}(\mathrm{Si}-\mathrm{O})$, the charge transfer effect can be
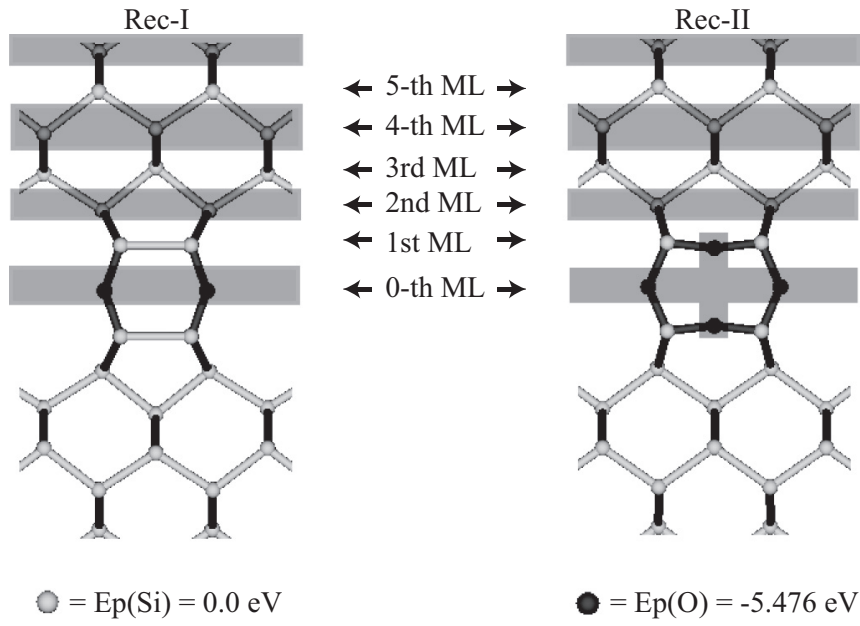

$=\mathrm{Ep}(\mathrm{Si})=0.0 \mathrm{eV}$ $=\frac{2 \mathrm{Vpp} \sigma(\mathrm{Si}-\mathrm{Si})+\mathrm{Vpp} \pi(\mathrm{Si}-\mathrm{Si})}{3}=-1.345 \mathrm{eV}$

尺 $=\mathrm{Vpp} \pi(\mathrm{Si}-\mathrm{Si})=-0.700 \mathrm{eV}$

$\mathrm{I}=\mathrm{Vpp} \pi(\mathrm{Si}-\mathrm{O})=-1.759 \mathrm{eV}$

FIG. 4. Schematic representations of our TB model. The spheres and bonds represent on-site and hopping matrix elements, respectively. The $i$ th MLs are defined by the gray and white regions.

ignored. Altogether, the effect of the coupling of the $p_{a}$ orbitals can be exclusively examined by our TB model. Note that the results obtained by using $V_{p p \pi}^{((\mathrm{I}))}(\mathrm{Si}-\mathrm{O})$ are shown below. The qualitatively same results are obtained by using $V_{p p \pi}^{(\mathrm{II})}(\mathrm{Si}-\mathrm{O})$.

Figure 5 shows that the VB1-top level of the Rec-II-20ML SL is the interface state, while the VB1-top WF of the Rec-I-20ML SL delocalizes over the Si layer. Since our TB model qualitatively reproduces the results from the $a b$ initio calculations, we conclude that the coupling of the $p_{a}$ orbitals induces the Rec-II interface state.

The interface hole state also occurs in a Si slab with $\mathrm{OH}-$ terminated Si-O-Si dimers [Fig. 3(c)]. However, the degree of localization is lowered in a $\mathrm{Si}$ slab with $\mathrm{H}$-terminated $\mathrm{Si}-\mathrm{O}$ Si dimers [Fig. 3(d)], in which less electrons are transferred from the surface $\mathrm{Si}$ atoms. However, when holes are doped (one electron per $\mathrm{Si}_{40} \mathrm{O}_{4} \mathrm{H}_{4}$ unit is removed), the degree of localization for the H-terminated Si slab increases [Fig. 3(e)]. These results indicate that the electron transfer induced by the bridging $\mathrm{O}$ atoms has a secondary effect on the formation of the Rec-II interface state. Moreover, our findings suggest that the locations of the channels of holes in a Si slab and a $\mathrm{Si}$ substrate also can be controlled by surface modification.

TABLE II. Parameters for our tight-binding model (in units of eV).

$\begin{array}{lr}E_{p}(\mathrm{Si}) & 0.000 \\ V_{p p \sigma}(\mathrm{Si}-\mathrm{Si}) & 2.367 \\ V_{p p \pi}(\mathrm{Si}-\mathrm{Si}) & -0.700 \\ E_{p}(\mathrm{O}) & -5.476 \\ V_{p p \pi}^{(\mathrm{I})}(\mathrm{Si}-\mathrm{O}) & -1.759 \\ V_{p p \pi}^{(\mathrm{II})}(\mathrm{Si}-\mathrm{O}) & -1.488\end{array}$




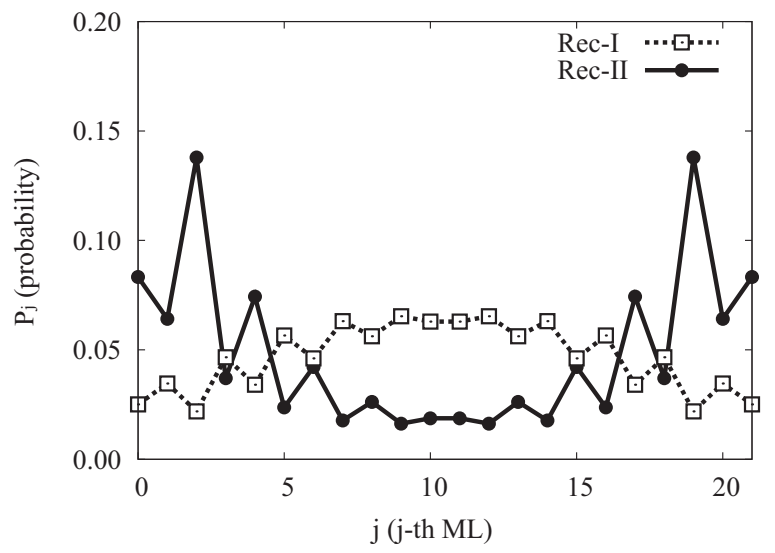

FIG. 5. Probability $\left(P_{j}\right)$ that an electron of the VB1-top state is found in the $j$ th ML. Here, $P_{j}=\sum_{i} l_{i j}\left|C_{i}\right|^{2} . l_{i j}=1$ if the atom $i$ is in the $j$ th ML, otherwise $l_{i j}=0 . C_{i}$ is the expansion coefficient for the $p_{a}$ orbital of the atom $i$. The zeroth and 21st MLs are O MLs. The other MLs are Si MLs. $P_{j}$ is normalized as $\sum_{j=0,20} P_{j}=1$.

In a previous $a b$ initio study [46], an interface hole state was found for a Si/quartz interface, but not found for a $\mathrm{Si} /$ cristobalite interface. From our findings, this result can be explained by the existence or nonexistence of Si-O-Si dimers at the $\mathrm{Si} / \mathrm{SiO}_{2}$ interface.

\section{Transport properties}

The $\mathrm{Si} / \mathrm{O} / \mathrm{Si}$ interface electron and hole states have the following three properties, which makes the Rec-I and Rec-II SLs attractive to be used for channel materials.

(i) The interface carriers have anisotropic effective masses, which is ideal for enhancing the carrier mobility along the channel ([110] direction) and reducing the gate-leakage current normal to the channel ([110] direction) as follows.

(a) The interface carriers have small effective masses along the [110] direction as shown in Table III, being ideal for enhancing the carrier mobility. Specifically, for the electrons in the Rec-I-4ML and Rec-I-20ML SLs, the effective masses of CB1 in the [110] direction $\left(m_{[110]}^{\mathrm{CB} 1}\right)$ are 0.12 and $0.14 m_{\mathrm{e}}$, respectively. Here, $m_{\mathrm{e}}$ is the electron

TABLE III. Properties of the electrons and holes in the epitaxial $\mathrm{Si} / \mathrm{O}$ SLs. The effective masses are given in units of electron mass. The energy splittings are given in units of $\mathrm{meV}$. For comparison purpose, the results for s-Si under $\mp 2 \mathrm{GPa}$ uniaxial stress in the [110] direction are also shown. For s-Si, $\Delta E_{\mathrm{CB}} \equiv\left|E_{\mathrm{CB} 3}-E_{\mathrm{CB} 1}\right|$ and $r_{[\overline{1} 10]}^{\mathrm{CB}} \equiv m_{[\overline{1} 10]}^{\mathrm{CB} 1} / m_{[\overline{1} 10]}^{\mathrm{CB} 3}$ because $\mathrm{CB} 1$ and $\mathrm{CB} 2$ are degenerated.

\begin{tabular}{lcccccc}
\hline \hline Electron & $m_{[110]}^{\mathrm{CB} 1}$ & $m_{[\overline{1} 10]}^{\mathrm{CB} 1}$ & $m_{[001]}^{\mathrm{CB} 1}$ & $\Delta E_{\mathrm{CB}}$ & $r_{[\overline{1} 10]}^{\mathrm{CB}}$ & $r_{[001]}^{\mathrm{CB}}$ \\
\hline Rec-I-4ML & 0.12 & 2.18 & 2.09 & 780 & 6.4 & 7.5 \\
Rec-I-20ML & 0.14 & 0.38 & 2.53 & 97 & 1.9 & 1.7 \\
s-Si (-2GPa) & 0.16 & 0.25 & 1.04 & 104 & 0.8 & 5.4 \\
hole & $m_{[110]}^{\mathrm{VB} 1}$ & $m_{[\overline{1} 10]}^{\mathrm{VB} 1}$ & $m_{[001]}^{\mathrm{VB} 1}$ & $\Delta E_{\mathrm{VB}}$ & $r_{[\overline{1} 110]}^{\mathrm{VB}}$ & $r_{[001]}^{\mathrm{VB}}$ \\
Rec-II-4ML & 0.09 & 1.82 & 0.74 & 801 & 3.6 & 1.2 \\
Rec-II-20ML & 0.11 & 2.88 & 0.40 & 132 & 1.2 & 6.4 \\
s-Si (+2GPa) & 0.11 & 1.90 & 0.27 & 115 & 15.0 & 1.2 \\
\hline \hline
\end{tabular}

mass. The calculated values are smaller than the electron effective mass obtained for strained $\mathrm{Si}$ (s-Si) under $2 \mathrm{GPa}$ uniaxial tensile stress in the [110] direction: $0.16 m_{\mathrm{e}}$. Similarly, for the holes in the Rec-II-4ML and Rec-II-20ML SLs, the values of $m_{[110]}^{\mathrm{VB} 1}$ are 0.09 and $0.11 m_{\mathrm{e}}$, respectively. These are smaller than or comparable to the hole effective mass of s-Si under $2 \mathrm{GPa}$ uniaxial compressive stress in the [110] direction: $0.11 \mathrm{~m}_{\mathrm{e}}$. The [110] direction is the best suited direction toward which the channel is oriented because both electrons and holes in the SLs take the smallest effective mass (see the Supplemental Material for details [30]).

(b) The interface carriers have large effective masses in the direction normal to the [110] direction, being ideal for reducing the gate-leakage current [13]. Specifically, for the electrons in the Rec-I-4ML (Rec-I-20ML) SL, $m_{[\overline{1} 10]}^{\mathrm{CB} 1}$ and $m_{[001]}^{\mathrm{CB} 1}$ are 2.18 and $2.09 m_{\mathrm{e}}\left(0.38\right.$ and $\left.2.53 m_{\mathrm{e}}\right)$, respectively. These are much larger than $m_{[110]}^{\mathrm{CB} 1}: 0.12 m_{\mathrm{e}}\left(0.14 m_{\mathrm{e}}\right)$. Similarly, for the holes in the Rec-II-4ML (Rec-II-20ML) SL, $m_{[\overline{1} 10]}^{\mathrm{VB} 1}$ and $m_{[001]}^{\mathrm{VB} 1}$ are 1.82 and $0.74 m_{\mathrm{e}}(2.88$ and $\left.0.40 m_{\mathrm{e}}\right)$, respectively. These are much larger than $m_{[110]}^{\mathrm{VB} 1}$ : $0.09 m_{\mathrm{e}}\left(0.11 m_{\mathrm{e}}\right)$.

(ii) Most electrons (holes) occupy the interface state, allowing us to make the best use of the small effective mass of the interface state, because the energy splitting $\Delta E_{\mathrm{CB}} \equiv\left|E_{\mathrm{CB} 2}-E_{\mathrm{CB} 1}\right|\left(\Delta E_{\mathrm{VB}} \equiv\left|E_{\mathrm{VB} 2}-E_{\mathrm{VB} 1}\right|\right)$ is much larger than $k_{\mathrm{B}} T$ at room temperature: $26 \mathrm{meV}$. Here, $E_{\mathrm{CB} i}$ $\left(E_{\mathrm{VB} i}\right)$ is the energy at the bottom of the $i$ th lowest conduction band $\mathrm{CB} i$ (top of the $i$ th highest valence band $\mathrm{VB} i$ ). $k_{\mathrm{B}}$ and $T$ are Boltzmann constant and room temperature $(300 \mathrm{~K})$, respectively. Furthermore, the value of $\Delta E_{\mathrm{CB}}\left(\Delta E_{\mathrm{VB}}\right)$ is expected to increase due to the quantum confinement when the gate bias is applied because $r_{[\overline{11} 10]}^{\mathrm{CB}}, r_{[001]}^{\mathrm{CB}}>1\left(r_{[\overline{11} 10]}^{\mathrm{VB}}, r_{[001]}^{\mathrm{VB}}>1\right)$ [13]. Here, $r_{[\overline{1} 10]}^{\mathrm{CB}} \equiv m_{[\overline{1} 10]}^{\mathrm{CB} 1} / m_{[\overline{1} 10]}^{\mathrm{CB} 2}$.

(iii) The interface states are nondegenerated, being ideal for reducing the interband scattering [12].

\section{E. Quantum confinement effects}

The localization of the electrons and holes around the interfaces is somehow unexpected and contrary to Tsu's expectations [22]. However, this does not imply that there is no quantum confinement effect in the Si/O SLs. Actually, the quantum confinement effects impact on the electronic states whose WFs delocalize over the Si layers. Interestingly, unlike for the nanostructures embedded in thick insulating layers, the impact of the quantum confinement induced by the $\mathrm{Si} / \mathrm{O} / \mathrm{Si}$ interfaces depends on the symmetry matching between the $\mathrm{WF}$ and the interface structure. The WFs of the $\mathrm{V}_{a}$ and $\mathrm{V}_{b}$ levels of the Rec-I-20ML SL are characterized by zigzag chains of $p$ orbitals along the $a$ and $b$ directions, respectively [Fig. 2(c)]. These two levels are degenerated in bulk Si. In the SL, however, the $\mathrm{V}_{a}$ level lies $94 \mathrm{meV}$ above the $\mathrm{V}_{b}$ level due to the symmetry-dependent quantum confinement effect. The $b$-directed chains do not match the five-membered rings of the interfaces. Therefore, the $\mathrm{V}_{b}$ WF cannot penetrate through the interface and is confined in the Si layers. On the other hand, the $a$-directed chains match the symmetry of the interface so that, although the amplitude is decreased 
around the interface, the $\mathrm{V}_{a} \mathrm{WF}$ penetrates through it, and the electronic states of the adjacent Si layers couple each other. Due to the coupling, the energy of the VB1, to which the $\mathrm{V}_{a}$ level belongs, increases as it goes from the $\Gamma$ point to the $Z$ point in the Brillouin zone.

For reference, we show the band structures of H-terminated 20ML-Si slabs in Appendix D. The band gaps $\left(E_{g} s\right)$ of Si nanostructures are usually higher than the $E_{g}$ of bulk $\mathrm{Si}$ due to quantum-confinement effects [1-10]. Actually, the $E_{g} \mathrm{~s}$ of the $20 \mathrm{ML} \mathrm{Si}$ slabs with H-terminated $\mathrm{Si}-\mathrm{Si}$ dimers (0.80 eV), OH-terminated Si-Si dimers (0.74 eV), $\mathrm{H}$-terminated nonreconstructed surface $(0.78 \mathrm{eV})$ are larger than the $E_{g}$ of bulk $\mathrm{Si}(0.62 \mathrm{eV}$ in our simulation scheme). Contrary to the conventional $\mathrm{Si}$ nanostructures, the $E_{g} \mathrm{~s}$ of the Rec-I-20ML SL $(0.63 \mathrm{eV})$ and the Rec-II-20ML SL $(0.50 \mathrm{eV})$ are comparable to and smaller than the $E_{g}$ of bulk $\mathrm{Si}$, respectively. This is because the interface states are not influenced by the quantum-confinement effects. Similarly, the $E_{g}$ of the $20 \mathrm{ML}$ Si slab with OH-terminated Si-O-Si dimers $(0.56 \mathrm{eV})$ is smaller than that of bulk Si. The $E_{g}$ of the 20ML $\mathrm{Si}$ slab with H-terminated Si-O-Si dimers $(0.64 \mathrm{eV})$ is larger than that of the $\mathrm{OH}$-terminated Si slab because of the weaker localization in the H-terminated Si slab. We also note that the Rec-I-20ML SL has a direct band gap. However, the radiative recombination rate, $41 \mathrm{~s}^{-1}$, is small because the WFs of the $\mathrm{C}_{\mathrm{I}}$ and $\mathrm{V}_{a}$ levels do not overlap well each other. For reference, the rates for $\mathrm{H}$-terminated $\mathrm{Si}$ nanodots of $2.4 \mathrm{~nm}$ in diameter [8] are more than $10^{3} \mathrm{~s}^{-1}$. (a) band structure

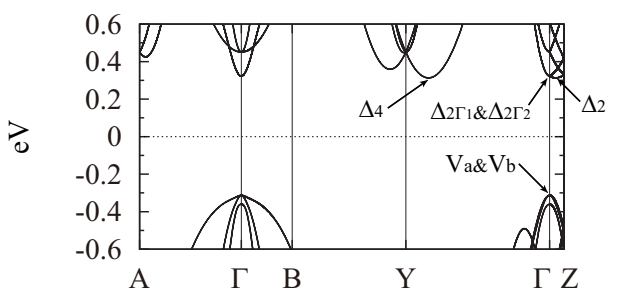

(c) zone folding

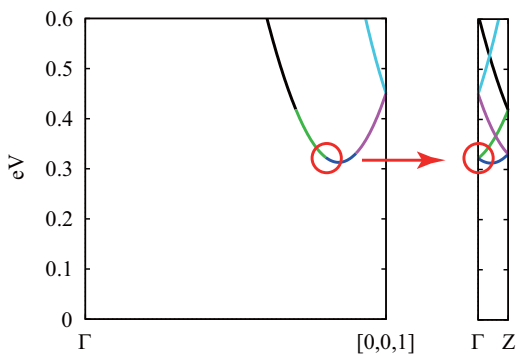

(b) unitcell

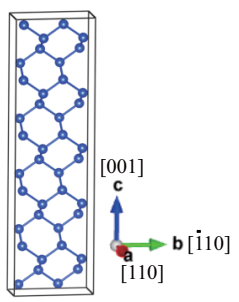

FIG. 6. (Color online) (a) Band structure of bulk Si calculated using a unit cell shown in (b). Note that $\mathrm{A}=\frac{2 \pi}{l_{\mathrm{b}}}\left(\frac{1}{2}, \frac{1}{2}, 0\right) \equiv\left[\frac{1}{2}, \frac{1}{2}, 0\right]$, $\Gamma=[0,0,0], \mathrm{B}=\left[-\frac{1}{4}, \frac{1}{4}, 0\right], \mathrm{Y}=[0,1,0]$, and $\mathrm{Z}=\left[0,0, \frac{1}{10}\right]$, where $l_{b}$ is the lattice constant of bulk Si. These points are not necessarily in the first Brillouin zone. (c) Zone folding. Left panel is the band structure of bulk Si calculated using the primitive unit cell shown in (d). Right panel is the band structure calculated using the unit cell shown in (b).

\section{F. Large valley splitting}

Another interesting consequence of the quantum confinement effect on the Rec-II-20ML SL consists in the energy splitting of the sixfold conduction band minimum of bulk $\mathrm{Si}$, being ideal for developing Si-based qubits [47]. Since the electron effective masses along the $z$ direction of the fourfold in-plane $\Delta_{4}$ valleys are smaller than those of the twofold out-of-plane $\Delta_{2}$ valleys, the increases in energy of the $\Delta_{4}$ valleys due to the quantum confinement are larger than those of the $\Delta_{2}$ valleys. The lower-energy $\Delta_{2}$ valleys further split into $\Delta_{2 \Gamma}$ and $\Delta_{2 Z}$ valleys due to the valley-valley coupling [47]. The splitting for Si nanostructures is usually small (at most $1 \mathrm{meV}$ [48]), which prevents developing Si-based qubits. For
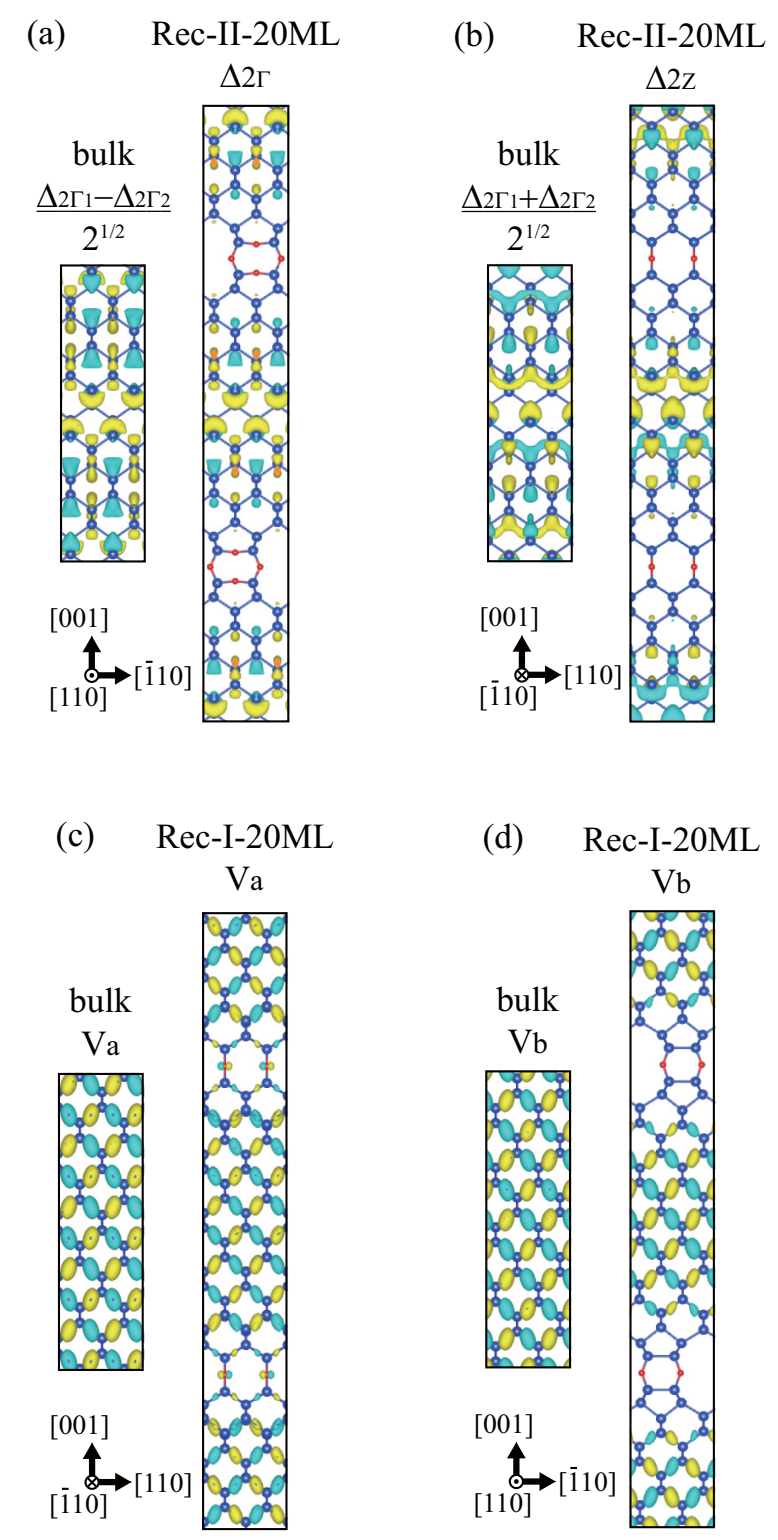

FIG. 7. (Color online) Comparison of WFs of bulk Si $(\Psi)$ and epitaxial Si/O SLs $(\Phi)$. Isosurfaces of (a) $\left(\Psi_{\Delta_{2 \Gamma_{1}}}-\Psi_{\Delta_{2 \Gamma_{2}}}\right) / 2^{1 / 2}$ and $\Phi_{\Delta_{2 \Gamma}}$, (b) $\left(\Psi_{\Delta_{2 \Gamma_{1}}}+\Psi_{\Delta_{2 \Gamma_{2}}}\right) / 2^{1 / 2}$ and $\Phi_{\Delta_{2 Z}}$, (c) $\Psi_{\mathrm{V}_{\mathrm{a}}}$ and $\Phi_{\mathrm{V}_{\mathrm{a}}}$, and (d) $\Psi_{\mathrm{V}_{\mathrm{b}}}$ and $\Phi_{\mathrm{V}_{\mathrm{b}}} . \pm 0.021$ isosurfaces for $\left(\Psi_{\Delta_{2 \Gamma_{1}}}-\Psi_{\Delta_{2 \Gamma_{2}}}\right) / 2^{1 / 2}$ and $\left(\Psi_{\Delta_{2 \Gamma_{1}}}+\Psi_{\Delta_{2 \Gamma_{2}}}\right) / 2^{1 / 2}, \pm 0.018$ for $\Phi_{\Delta_{2 \Gamma}}$ and $\Phi_{\Delta_{2 Z}}, \pm 0.020$ for $\Psi_{\mathrm{V}_{\mathrm{a}}}, \Psi_{\mathrm{V}_{\mathrm{b}}}, \Phi_{\mathrm{V}_{\mathrm{a}}}$, and $\Phi_{\mathrm{V}_{\mathrm{b}}}$ are shown. 
the Rec-II-20ML SL, however, the magnitude of the energy splitting is $15.3 \mathrm{meV}$ (see the Supplemental Material for the accuracy [30]). This value is larger than the reported values for a 20ML Si well embedded in $\mathrm{Ge}(2 \mathrm{meV})$ and for a 40ML $\mathrm{Si}$ well embedded in an optimal Si/Ge SL (8.7 meV) [47].

We also investigate the valley splitting in Si slabs. The values for the $20 \mathrm{ML} \mathrm{Si}$ slabs with $\mathrm{OH}$-terminated $\mathrm{Si}-\mathrm{O}-\mathrm{Si}$ dimers (12.2 meV), H-terminated Si-O-Si dimers (11.0 meV), and $\mathrm{OH}$-terminated $\mathrm{Si}-\mathrm{Si}$ dimers $(11.8 \mathrm{meV})$ are larger than those for $\mathrm{H}$-terminated $\mathrm{Si}-\mathrm{Si}$ dimers $(2.1 \mathrm{meV})$ and $\mathrm{H}$ terminated nonreconstructed surface $(0.1 \mathrm{meV})$. These results imply that the surface oxidation enhances the valley splitting. The formation of $\mathrm{Si}-\mathrm{O}$ bonds near $\mathrm{Si} / \mathrm{SiO}_{2}$ interfaces might play an important role for the 23-meV large splitting observed in $\mathrm{a} \mathrm{SiO}_{2} / \mathrm{Si}(001) / \mathrm{SiO}_{2}$ quantum well [49].

\section{CONCLUSION}

To conclude, using geometrical considerations and $a b$ initio calculations, we have shown that our Rec-I and Rec-II Si/O SL models are energetically more favorable than all the models proposed so far. Our $\mathrm{Si} / \mathrm{O} / \mathrm{Si}$ interface models are composed of reconstructed $\mathrm{Si}\{001\}$ surfaces connected by $\mathrm{Si}-\mathrm{O}-\mathrm{Si}$ bridges, which allows each atom to maintain its natural

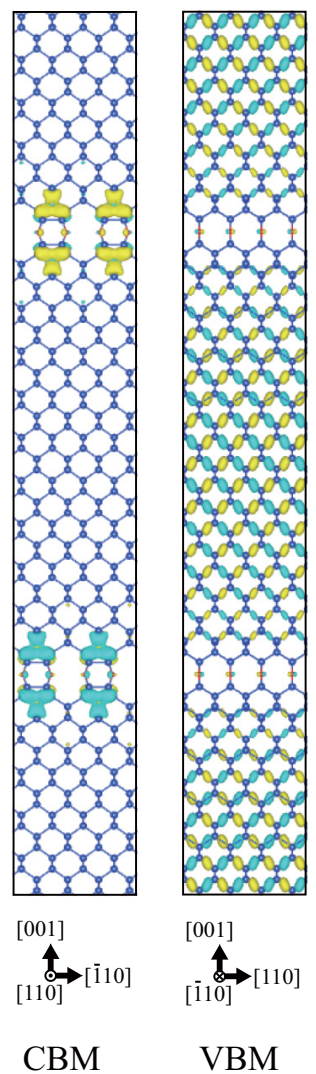

(a) Rec-I-40ML

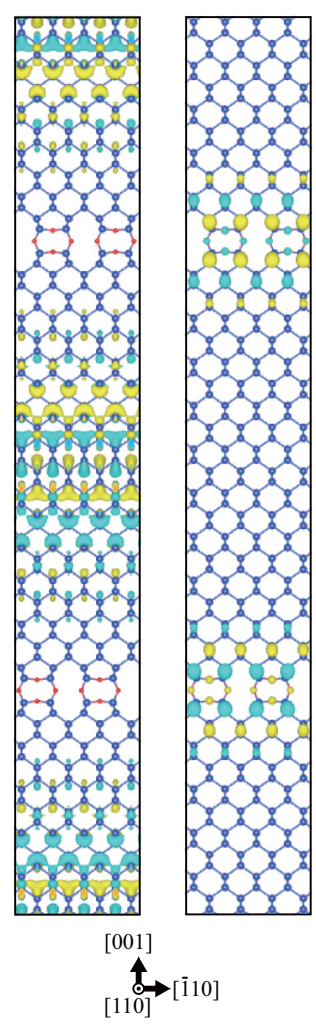

CBM VBM
FIG. 8. (Color online) Isosurfaces of CBM and VBM WFs. (a) Epitaxial Rec-I-40ML superlattice. \pm 0.020 and $\pm 0.020 / 2^{1 / 2}$ isosurfaces are shown for the CBM and VBM WFs, respectively. (b) Epitaxial Rec-II-40ML superlattice. $\pm 0.018 / 2^{1 / 2}$ and \pm 0.020 isosurfaces are shown for the CBM and VBM WFs, respectively. bonding configuration. The part of the Rec-II interface is experimentally accessible just by oxidizing a $\mathrm{Si}(001)$ substrate. To complete the Rec-II superlattice, we have proposed a three-step method. We have also explored the potential of our $\mathrm{Si} / \mathrm{O}$ superlattice models for new materials used in future $\mathrm{Si}$ electronics. To our surprise, the Rec-I and Rec-II interfaces create the interface electron and hole states, respectively. The Rec-I interface state originates from the interactions between the adjacent Si layers. This indicates that similar interface states should be easily realized experimentally just by facing H-terminated reconstructed $\mathrm{Si}(001)$ substrates toward each other or by stacking Si slabs with H-terminated Si-Si dimers. On the other hand, the $p_{a}$ orbitals of $\mathrm{O}$ atoms inserted in the $\mathrm{Si}$-Si dimers induce the Rec-II interface state. This indicates that the locations of channels of holes in a $\mathrm{Si}$ slab and a $\mathrm{Si}$ substrate can be controlled by surface modification as well. Interestingly, the Rec-I and Rec-II interface carriers have small effective masses in the direction parallel to the channel ([110] direction) and large effective masses in the direction normal to the channel ([110] direction), making the $\mathrm{Si} / \mathrm{O}$ superlattices attractive to be used for channel materials. We have also shown that the valley splitting of $\mathrm{Si}$ is enhanced by the formation of the $\mathrm{Si} / \mathrm{O} / \mathrm{Si}$ interfaces, being ideal for developing $\mathrm{Si}$ based qubits. Our findings open new perspectives to design and control the electronic properties of Si.

\section{ACKNOWLEDGMENTS}

This work is supported by AIST-IMEC collaboration project. K.N. wishes to thank T. Ozaki, N. Orita, and S. Ishibashi for fruitful discussions. GDIS [50] was used to generate the atomic arrangements. VESTA [51] was used to illustrate isosurfaces of WFs.

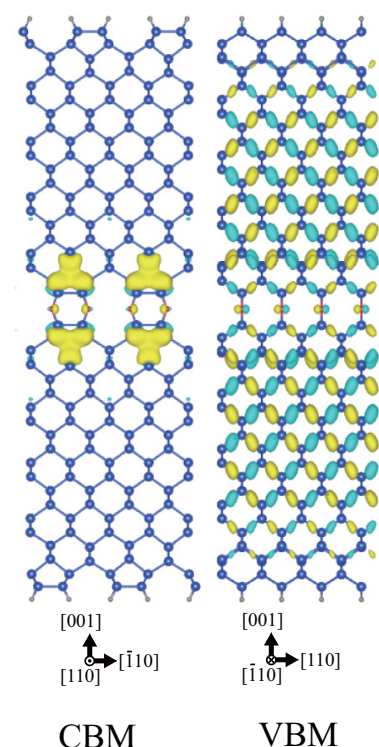

(a) Rec-I slab

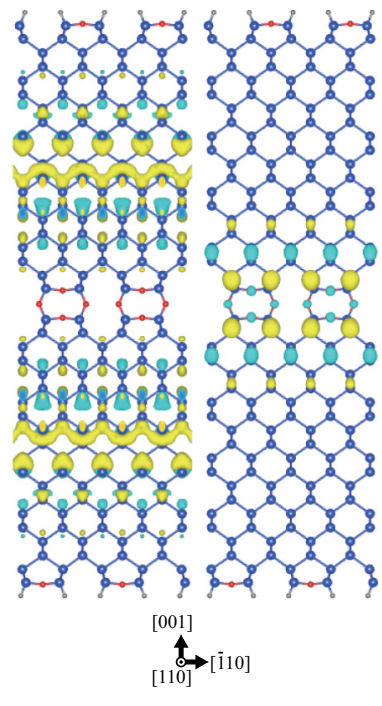

CBM VBM
FIG. 9. (Color online) Isosurfaces of CBM and VBM WFs. (a) Rec-I slab. $\pm 0.020 \times 2^{1 / 2}$ and \pm 0.020 isosurfaces are shown for the CBM and VBM WFs, respectively. (b) Rec-II slab. \pm 0.018 and $\pm 0.020 \times 2^{1 / 2}$ isosurfaces are shown for the CBM and VBM WFs, respectively. 


\section{APPENDIX A: BAND STRUCTURE AND WAVE FUNCTIONS OF BULK SI}

The band structure of bulk Si calculated using a unit cell containing $20 \mathrm{ML}$ of Si is shown in Fig. 6(a). The conduction band minimum of bulk $\mathrm{Si}$ is sixfold, namely, the $\Delta_{2}$ and $\Delta_{4}$ levels are degenerated. The $\Delta_{2 \Gamma_{1}}$ and $\Delta_{2 \Gamma_{2}}$ levels, which are the twofold lowest energy levels of the conduction band at $\Gamma$, are folded from the $\frac{2 \pi}{l_{b}}\left(0,0, \pm \frac{8}{10}\right)$ points of the band structure calculated using the primitive unit cell [Figs. 6(c) and 6(d)].

Figures 7(a) and 7(b) show that the $\Delta_{2 \Gamma}$ and $\Delta_{2 Z}$ levels of the Rec-II-20ML SL originate from the $\Delta_{2 \Gamma_{1}}$ and $\Delta_{2 \Gamma_{2}}$ levels of bulk Si, respectively. Actually, $\Phi_{\Delta_{2 \Gamma}}$ and $\Phi_{\Delta_{2 Z}}$ resemble to $\left(\Psi_{\Delta_{2 \Gamma_{1}}}-\Psi_{\Delta_{2 \Gamma_{2}}}\right) / 2^{1 / 2}$ and $\left(\Psi_{\Delta_{2 \Gamma_{1}}}+\Psi_{\Delta_{2 \Gamma_{2}}}\right) / 2^{1 / 2}$, respectively. Here, $\Phi_{\Delta_{2 \Gamma}}\left(\Phi_{\Delta_{2 Z}}\right)$ is the WF of the $\Delta_{2 \Gamma}\left(\Delta_{2 Z}\right)$ level of the Rec-II-20ML SL and $\Psi_{\Delta_{2 \Gamma_{1}}}\left(\Psi_{\Delta_{2 \Gamma_{2}}}\right)$ is the WF of the $\Delta_{2 \Gamma_{1}}$ $\left(\Delta_{2 \Gamma_{2}}\right)$ level of bulk Si. Note that the molecular orbitals of the adjacent Si layers in the $\Phi_{\Delta_{2 \Gamma}}$ are in phase because the $\Delta_{2 \Gamma}$ level is located at the $\Gamma$ point. On the other hand, the molecular orbitals of the adjacent Si layers in the $\Phi_{\Delta_{2 Z}}$ are out of phase because the $\Delta_{2 Z}$ level is located at the $Z$ point. Figures 7(c) and 7(d) also show that the $V_{a}$ and $V_{b}$ levels of the Rec-I-20ML SL originate from the $\mathrm{V}_{a}$ and $\mathrm{V}_{b}$ levels of bulk $\mathrm{Si}$, respectively.

(a) H-terminated non-reconstructed surface

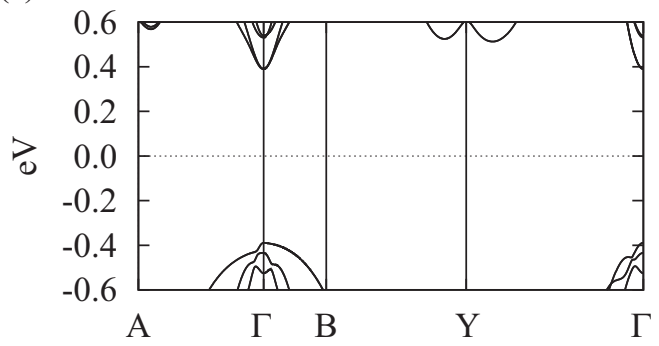

(c) OH-terminated Si-Si dimers

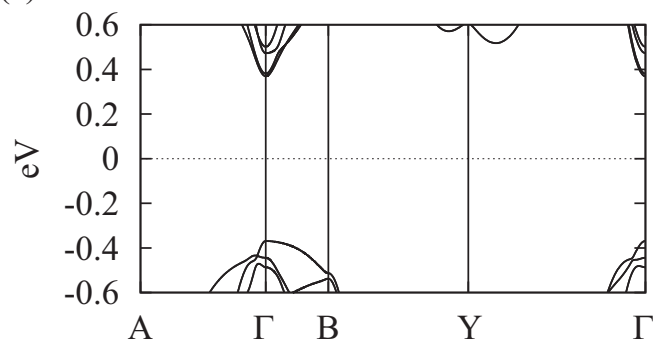

(e) H-terminated Si-O-Si dimers

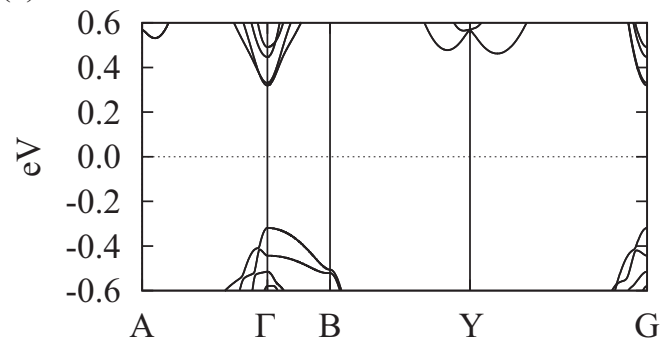

\section{APPENDIX B: WAVE FUNCTIONS OF THE REC-I-AND REC-II-40ML SUPERLATTICES}

The WFs of the epitaxial Rec-I- and Rec-II-40ML SLs were calculated using Si7.0-s $2 p 2 d 1$ and O5.0-s $2 p 2 d 1$ basis sets, and are shown in Fig. 8. The atomic positions are optimized using an out-of-plane unit-cell vector $c=\left(-\frac{l_{b}}{4}, \frac{l_{b}}{4}, c_{z 20 \mathrm{ML}}+\right.$ $\left.5 l_{b}\right)$. Here, $c_{z 20 \mathrm{ML}}$ is the calculated $z$ component of $c$ for the corresponding $20 \mathrm{ML}$ SL.

The conduction-band-minimum (CBM) WF of the Rec-I40ML SL is localized around the Rec-I interface, while the valence-band-maximum (VBM) WF delocalizes over the Si layers. For the Rec-II-40ML SL, reversely, the VBM WF is localized around the Rec-II interface, while the CBM WF delocalizes over the Si layers.

\section{APPENDIX C: WAVE FUNCTIONS OF SLAB MODELS HAVING ONE SI/O/Si INTERFACE}

The WFs of isolated slabs composed of one $\mathrm{Si} / \mathrm{O} / \mathrm{Si}$ interface between two Si layers are shown in Fig. 9. Each Si layer consists of $20 \mathrm{MLs}$ of Si. The surface dangling bonds are terminated by hydrogen to remove the dangling-bond states from the band gap. The $\mathrm{H}$ atoms are placed $1.4 \AA$ apart from surface $\mathrm{Si}$ atoms, but the atomic positions are not optimized.

(b) H-terminated Si-Si dimers

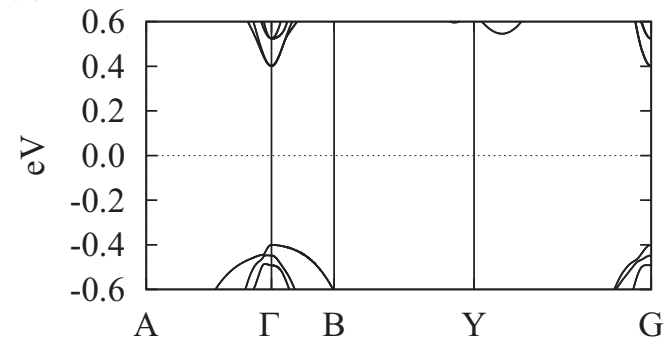

(d) hole-doped H-terminated Si-Si dimers

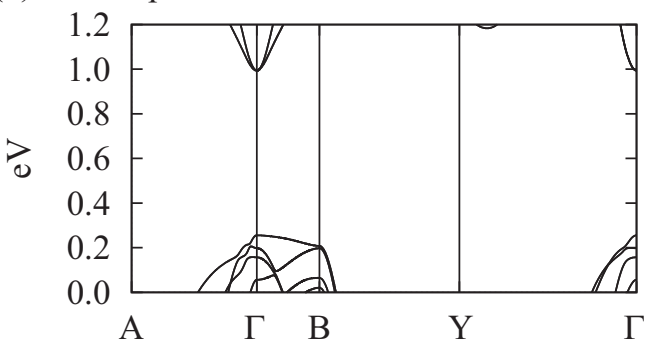

(f) $\mathrm{OH}$-terminated Si-O-Si dimers

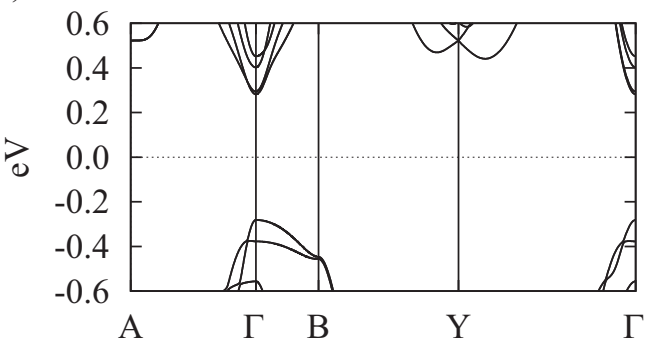

FIG. 10. Band structures of 20ML Si slabs with (a) H-terminated non-reconstructed surface, (b) H-terminated Si-Si dimers, (c) OHterminated $\mathrm{Si}$-Si dimers, (d) hole-doped H-terminated $\mathrm{Si}$-Si dimers: one electron per $\mathrm{Si}_{40} \mathrm{O}_{4} \mathrm{H}_{4}$ unit is removed, (e) H-terminated $\mathrm{Si}$-O-Si dimers, and (f) OH-terminated Si-O-Si dimers. 
Note that, in Appendix D, the $\mathrm{H}$ atoms are placed $1.48 \AA$ apart from surface $\mathrm{Si}$ atoms, but the small difference in $\mathrm{Si}-\mathrm{H}$ bond length does not affect the results.

The CBM WF of the Rec-I slab is localized around the Rec-I interface, while the VBM WF delocalizes over the Si layers. For the Rec-II slab, reversely, the VBM WF is localized around the Rec-II interface, while the CBM WF delocalizes over the $\mathrm{Si}$ layers. These results show that the formation of one $\mathrm{Si} / \mathrm{O} / \mathrm{Si}$ interface is enough to create the interface channel.

\section{APPENDIX D: BAND STRUCTURES OF H-TERMINATED 20 ML SI SLABS}

The band structures of 20ML Si slabs are shown in Fig. 10. The structure models are constructed by cutting out bulk Si, Rec-I-20ML SL, or Rec-II-20ML SL. The H atoms are placed 1.48 and $1.00 \AA$ apart from surface $\mathrm{Si}$ and $\mathrm{O}$ atoms, respectively. Note that each surface $\mathrm{Si}$ atom of nonreconstructed surface has two $\mathrm{Si}-\mathrm{H}$ bonds.
[1] H. Takagi, H. Ogawa, Y. Yamazaki, A. Ishizaki, and T. Nakagiri, Appl. Phys. Lett. 56, 2379 (1990).

[2] L. T. Canham, Appl. Phys. Lett. 57, 1046 (1990).

[3] A. G. Cullis, L. T. Canham, and P. D. J. Calcott, J. Appl. Phys. 82, 909 (1997).

[4] D. J. Lockwood, Z. H. Lu, and J.-M. Baribeau, Phys. Rev. Lett. 76, 539 (1996).

[5] Z. H. Lu and D. Grozea, Appl. Phys. Lett. 80, 255 (2002).

[6] N. Tit and M. Dharma-wardana, Solid State Commun. 106, 121 (1998).

[7] P. Carrier, L. J. Lewis, and M. W. C. Dharma-wardana, Phys. Rev. B 65, 165339 (2002).

[8] K. Nishio, J. Kōga, T. Yamaguchi, and F. Yonezawa, Phys. Rev. B 67, 195304 (2003).

[9] Y. Kanemitsu, Phys. Rep. 263, 1 (1995).

[10] H. Kageshima, Surf. Sci. 357-358, 312 (1996).

[11] C. S. Smith, Phys. Rev. 94, 42 (1954).

[12] D. K. Nayak and S. K. Chun, Appl. Phys. Lett. 64, 2514 (1994).

[13] S. Thompson, G. Sun, Y. S. Choi, and T. Nishida, IEEE Trans. Electron Devices 53, 1010 (2006).

[14] Z. Liu, J. Wu, W. Duan, M. G. Lagally, and F. Liu, Phys. Rev. Lett. 105, 016802 (2010).

[15] M. Zhou, Z. Liu, Z. Wang, Z. Bai, Y. Feng, M. G. Lagally, and F. Liu, Phys. Rev. Lett. 111, 246801 (2013).

[16] M. Fornari, Physics 6, 140 (2013).

[17] G. L. W. Hart, S. Curtarolo, T. B. Massalski, and O. Levy, Phys. Rev. X 3, 041035 (2013).

[18] K. Koga, G. T. Gao, H. Tanaka, and X. C. Zeng, Nature (London) 412, 802 (2001).

[19] K. Takeda and K. Shiraishi, Phys. Rev. B 50, 14916 (1994).

[20] P. Vogt, P. De Padova, C. Quaresima, J. Avila, E. Frantzeskakis, M. C. Asensio, A. Resta, B. Ealet, and G. Le Lay, Phys. Rev. Lett. 108, 155501 (2012).

[21] A. Fleurence, R. Friedlein, T. Ozaki, H. Kawai, Y. Wang, and Y. Yamada-Takamura, Phys. Rev. Lett. 108, 245501 (2012).

[22] R. Tsu, Nature (London) 364, 19 (1993).

[23] R. Tsu and J. C. Lofgren, J. Cryst. Growth 227-228, 21 (2001).

[24] K. Dovidenko, J. Lofgren, F. de Freitas, Y. Seo, and R. Tsu, Physica E 16, 509 (2003).

[25] R. J. Mears, M. Hytha, I. Dukovski, A. Yiptong, X. Huang, S. Halilov, A. Broka, R. J. Stephenson, V. Rao, D. R. Webb, R. Prasad, S. A. Kreps, H. Takeuchi, S. Ikeda, G. Gebara, K. Matthews, J. Wetzel, W. Xiong, C. Bowen, R. Wise, and C. R. Cleavelin, in Proceedings of the IEEE International SOI Conference (IEEE, New York, 2007), pp. 23-24.

[26] A. Delabie, S. Jayachandran, M. Caymax, R. Loo, J. Maggen, G. Pourtois, B. Douhard, T. Conard, J. Meersschaut, H. Lenka,
W. Vandervorst, and M. Heyns, ECS Solid State Lett. 2, P104 (2013).

[27] X. Luo, S. B. Zhang, and S.-H. Wei, Phys. Rev. Lett. 89, 076802 (2002).

[28] R. M. Tromp, R. J. Hamers, and J. E. Demuth, Phys. Rev. Lett. 55, 1303 (1985).

[29] Y. Tu and J. Tersoff, Phys. Rev. Lett. 84, 4393 (2000).

[30] See Supplemental Material at http://link.aps.org/supplemental/ 10.1103/PhysRevB.91.165303 for details in geometry optimization, accuracy of valley-splitting energy, and anisotropic effective masses.

[31] http://qmas.jp/.

[32] J. P. Perdew, K. Burke, and M. Ernzerhof, Phys. Rev. Lett. 77, 3865 (1996).

[33] P. E. Blöchl, Phys. Rev. B 50, 17953 (1994).

[34] A. Ourmazd, D. W. Taylor, J. A. Rentschler, and J. Bevk, Phys. Rev. Lett. 59, 213 (1987).

[35] A. Pasquarello, M. S. Hybertsen, and R. Car, Phys. Rev. Lett. 74, 1024 (1995).

[36] T. Yamasaki, C. Kaneta, T. Uchiyama, T. Uda, and K. Terakura, Phys. Rev. B 63, 115314 (2001).

[37] H. Kageshima, M. Uematsu, K. Akagi, S. Tsuneyuki, T. Akiyama, and K. Shiraishi, e-J. Surf. Sci. Nanotech. 4, 584 (2006).

[38] N. Ikarashi, K. Watanabe, and Y. Miyamoto, Phys. Rev. B 62, 15989 (2000).

[39] M. M. Roberts, L. J. Klein, D. E. Savage, K. A. Slinker, M. Friesen, G. Celler, M. A. Eriksson, and M. G. Lagally, Nature Mater. 5, 388 (2006).

[40] http://www.openmx-square.org/

[41] N. Troullier and J. L. Martins, Phys. Rev. B 43, 1993 (1991).

[42] A. H. MacDonald and S. H. Vosko, J. Phys. C 12, 2977 (1979).

[43] G. B. Bachelet, D. R. Hamann, and M. Schlüter, Phys. Rev. B 26, 4199 (1982).

[44] G. Theurich and N. A. Hill, Phys. Rev. B 64, 073106 (2001).

[45] W. A. Harrison and Physics, Electronic Structure and the Properties of Solids: The Physics of the Chemical Bond (Dover Publications, New York, 1989).

[46] Y. Suwa and S.-i. Saito, Phys. Rev. B 79, 233308 (2009).

[47] L. Zhang, J.-W. Luo, A. Saraiva, B. Koiller, and A. Zunger, Nature Commun. 4, 2396 (2013).

[48] F. Zwanenburg, A. Dzurak, A. Morello, M. Simmons, L. Hollenberg, G. Klimeck, S. Rogge, S. Coppersmith, and M. Eriksson, Rev. Mod. Phys. 85, 961 (2013).

[49] K. Takashina, Y. Ono, A. Fujiwara, Y. Takahashi, and Y. Hirayama, Phys. Rev. Lett. 96, 236801 (2006).

[50] http://gdis.sourceforge.net/index.html/.

[51] K. Momma and F. Izumi, J. Appl. Crystallogr. 44, 1272 (2011). 